\title{
Más allá de los cuerpos dóciles. El teatro de Eve Ensler como proyecto 'boaliano'
}

\author{
Marta Fernández Morales \\ Universitat de les Illes Balears \\ marta.fernandez@uib.es \\ https://dx.doi.org/10.12795/futhark.2012.i07.05
}

\begin{abstract}
In an interview that was published in 2001, U.S-born playwright Eve Ensler stated that her mission as an author and performer was to raise the consciousness of her audience about atrocity and injustice. Most of her plays, including the well-known and often staged The Vagina Monologues (1998), are devoted to the denunciation of atrocity and injustice as they are inscribed on the female body. In Ensler's production, women and girls are placed at the narrative and dramaturgical centre, and their bodies become the source of anger and rage, but also of self-knowledge, rebellion, pleasure, and sisterhood. Within a potentially Boalian framework which intends to transform the audience, encouraging it to assume the role of an agent, Ensler articulates proposals that give voice to the female body as sexed cultural matter, in the line of The Good Body (2001), Fur Is Back (2007), and I Am an Emotional Creature (2010). My objective here will be to try and prove that Ensler's theatrical praxis has a place within Augusto Boal's (19312009) universe of the Theatre of the Oppressed, and that her work is also developed around the aim of overcoming the Foucaultian concept of a 'docile body', urging girls and women to empower themselves precisely from a locus that the dominant culture has tried to objectify and control through its discursive practices: their body.
\end{abstract}

Keywords: Theatre. Body. Consciousness-raising. Agency. Empowerment.

Resumen: La dramaturga estadounidense Eve Ensler declaró en una entrevista publicada en 2001 que su misión como autora y performer era despertar la conciencia de su público sobre la atrocidad y la injusticia. La mayoría de sus trabajos, entre ellos la conocida y muy representada pieza The Vagina Monologues (1998), se ha dedicado a denunciar esa atrocidad y esa injusticia en sus formas de inscripción sobre los cuerpos femeninos. En toda la producción de Ensler las mujeres y las niñas son el epicentro narrativo y dramatúrgico, y sus cuerpos se convierten en fuentes de ira y de rabia, pero también de autoconocimiento, rebeldía, placer y sororidad. Dentro de un marco potencialmente 'boaliano' que pretende transformar al público espectador moviéndole a asumir un rol de agente, Ensler articula propuestas que dan voz al cuerpo femenino como materia cultural sexuada, en la línea de The Good Body ("El buen cuerpo", 2001), Fur Is Back ("Vuelven las pieles", 2007) o I Am an Emotional Creature ("Soy una criatura emocional", 2010). El objetivo aquí es demostrar que la praxis teatral de Ensler tiene cabida en el universo de Augusto Boal (1931-2009), creador del Teatro de los Oprimidos, y que, además, gira en torno al objetivo de superar Futhark 7 (2012)

Recibido 02/05/2011 ISSN 1886-9300

Aceptado 30/06/2011 
el concepto foucaultiano de organismo dócil, invitando a niñas y mujeres a empoderarse a partir de aquello que la cultura dominante ha querido reificar y controlar a través de sus prácticas discursivas: el cuerpo.

Palabras clave: Teatro. Cuerpo. Toma de conciencia. Agencia. Empoderamiento.

\section{Introducción: Contra la injusticia, teatro}

La dramaturga estadounidense Eve Ensler (Nueva York, 1953) declaró en una entrevista con la crítica teatral Alexis Greene publicada en 2001 que su misión principal como autora y performer era despertar la conciencia de su público sobre la atrocidad y la injusticia ${ }^{1}$. De hecho, todos sus trabajos desde la primera edición de la ya bastante conocida y muy representada pieza The Vagina Monologues $(1998)^{2}$ se han dedicado precisamente a denunciar esa atrocidad y esa injusticia en sus formas de inscripción sobre los cuerpos femeninos y, por extensión, sobre la vida de ciudadanas de todo el mundo. En la obra de Ensler las mujeres y las niñas son el epicentro narrativo y dramatúrgico, y sus cuerpos se convierten en fuentes de lo bueno y de lo malo: son el punto de partida para el autoconocimiento, el origen de la rebeldía y el foco del placer; pero son también el lugar hacia el que se dirige la violencia, del que nace la ira y en el que anida la rabia. Dentro de un marco potencialmente 'boaliano' que, como se explicará aquí, pretende transformar al público espectador moviéndole a asumir un rol de agente en el teatro y en la vida, Ensler articula propuestas centradas en el cuerpo femenino como materia cultural sexuada, en la línea de obras dramáticas como la agridulce The Good Body (2001), la intensa Fur Is Back (2007) o la más reciente I Am an Emotional Creature (2010). Enfocando mi objeto de estudio desde la perspectiva de género y partiendo de los textos primarios y de las ideas de la propia Ensler respecto a la función del teatro, mi finalidad aquí será tratar de demostrar que su actividad dramatúrgica tiene cabida en el universo del Teatro de los Oprimidos de Augusto Boal y que, además, gira en torno al objetivo de superar el concepto foucaultiano de organismo dócil, invitando a niñas y mujeres a empoderarse precisamente a

\footnotetext{
${ }^{1}$ GreENE, Alexis, Women Who Write Plays. Interviews with American Dramatists, Hanover, Smith \& Kraus, 2001, pág. 165.

${ }^{2}$ Traducida como Los monólogos de la vagina y publicada por Planeta en 2000. Hasta la fecha, es el único trabajo de Ensler que ha sido editado en español.

Futhark 7 (2012) $\quad$ Fernández, Más allá de los cuerpos dóciles, 131-161
}

ISSN $1886-9300$ 
partir de aquello que la cultura dominante ha querido siempre reificar y controlar a través de sus prácticas discursivas: el cuerpo.

La primera vez que Eve Ensler puso en escena The Vagina Monologues -en Nueva York en el año 1994- sólo unos pocos cientos de personas vieron la función, pero su impacto más allá del éxito comercial es destacable, ya que con aquel montaje se encendió una llama transformadora que aún continúa ardiendo: el Día- $V^{3}$, cuyo nacimiento oficial se fecha en 1998 y que se define, según su portal en Internet en español, como "un movimiento global de activistas que trabaja para detener la violencia contra mujeres y niñas". El activismo del Día-V se articula alrededor de la creación artística, ya que la iniciativa nació de la puesta en escena de The Vagina Monologues y ahora incluye otras obras dramáticas de Ensler, así como documentales, concursos de ideas para campañas contra la agresión machista y un libro de fotografías de Joyce Tenneson titulado Vagina Warriors (2005), entre otros materiales. En los años que lleva funcionando la Fundación Día- $V$, la dramaturga y su creciente equipo de "guerreras y guerreros de la vagina" han conseguido llegar a más de 130 países, con los fines declarados de promover la creatividad para remover las conciencias, recaudar dinero para revitalizar organizaciones anti-violencia (más de 80 millones de dólares hasta el día de hoy) y llamar la atención sobre distintas manifestaciones de la violencia de género ${ }^{4}$. El movimiento toma diferentes formas en varias campañas según el público al que se dirija, incluyendo Día-V Campus (en las universidades), Chicas-V (trabajando con adolescentes y jóvenes), Hombres-V (tratando específicamente cuestiones de masculinidad) y Día-V Foco (centrándose en una manifestación concreta de la agresión sexista). Entre las temáticas que se han tocado hasta ahora en la línea spotlight ("foco", asunto en el que se centraliza la atención cada año), están, por ejemplo, la situación de las mujeres en Afganistán, donde el conflicto político-religioso y las ideas de los talibán siguen haciendo estragos; las formas de violencia sufridas por las

\footnotetext{
${ }^{3}$ Para un análisis más detallado del Día-V como movimiento artístico y social, véase mi artículo "When Theater Becomes a Crusade against Violence", en BAHUNRadunOvić, Sanja; RAJAN, V.G. Julie (eds.), Violence and Gender in the Globalizad World. The Intimate and the Extimate, Aldershot y Burlington: Ashgate, 2008, págs. 205-221.

${ }^{4}<$ www.vday.org>. Fecha de acceso: 19 de abril de 2011.
}

Futhark 7 (2012) Fernández, Más allá de los cuerpos dóciles, 131-161

ISSN $1886-9300$ 
ciudadanas nativoamericanas en Estados Unidos (los índices de maltrato doméstico en las reservas indias son especialmente graves); las muertas y desaparecidas de Ciudad Juárez, sepultadas bajo el manto del silencio y la impunidad por la inacción de las autoridades; la experiencia de las iraquíes durante el último conflicto armado; la exigencia de reconocimiento y compensación para las orientales prostituidas de la $2^{\mathrm{a}}$ Guerra Mundial y la vida de las habitantes de lugares como Nueva Orleáns, el Congo o Haití, todas ellas afectadas por formas de agresión marcadas por el género que se suman a la violencia estructural que padecen sus comunidades y que se acentúa debido a las crisis políticas, económicas, naturales y/o humanitarias que viven en la actualidad. En algunos de estos sitios la Fundación Día-V ha llegado a abrir refugios y centros comunitarios donde las mujeres y las niñas pueden sentirse seguras, ir a formarse para trabajar, curarse de enfermedades como la fístula obstétrica o trabajar su posición de líderes en sus respectivas sociedades ${ }^{5}$. En todos ellos, además, se ha puesto en escena The Vagina Monologues, a veces incluso en la clandestinidad y en lugares inesperados. Los espacios en los que han tenido y tienen lugar los eventos teatrales del Día-V incluyen escenarios tan dispares como teatros, iglesias, estadios de fútbol, universidades o clubes nocturnos, entre otros. Ello conecta con la idea defendida por Boal dentro del marco del Teatro de los Oprimidos de que la actividad dramática puede darse allí donde el público sea capaz de concebir un "espacio estético". El autor brasileño definía ese espacio como el lugar donde la mirada de los espectadores y espectadoras encuadra los procesos que están llevando a cabo los/as intérpretes y donde las acciones adquieren nuevas propiedades, los objetos dejan de tener su significado cotidiano para convertirse en signos con nuevos significados, y cada gesto se magnifica. El espacio estético tenía para Boal cinco dimensiones: las tres físicas, más las dimensiones subjetivas de la imaginación y la memoria ${ }^{6}$, ya que es el resultado de un proceso creador subjetivo por parte del público ${ }^{7}$.

\footnotetext{
${ }^{5}$ Un listado actualizado de los refugios que van poniéndose en marcha por iniciativa del Día-V se puede encontrar en <www.vday.org/our-work/safe-houses>.

${ }^{6}$ BOAL, Augusto, Legislative Theatre. Using Performance to Make Politics, Londres y Nueva York, Routledge, 2005 (1998), pag. 72.

${ }^{7}$ BoAL, Augusto, The Rainbow of Desire. The Boal Method of Theatre and Therapy, Londres y Nueva York: Routledge, 2006a (1995), pág. 18.

Futhark 7 (2012) Fernández, Más allá de los cuerpos dóciles, 131-161
}

ISSN 1886-9300 
Al incorporar las experiencias de mujeres de distintos países y culturas al texto y los eventos generados alrededor de The Vagina Monologues -una pieza siempre viva y en proceso de reescritura, como recoge Kattwinkel en su discusión sobre la corrección política y la 'americanidad' de la obra- ${ }^{8}$, así como a otras obras, y al hacer el esfuerzo añadido de visibilizar sus problemas a través de charlas, películas, páginas web, talleres, trabajo sobre el terreno, etc., Eve Ensler y el Día-V consiguen romper el silencio sobre temas espinosos u olvidados, sembrar el desasosiego y, a menudo, la necesidad de actuar entre quienes participan en los eventos de la iniciativa de una u otra manera. Se trata, como han comentado las productoras de The Vagina Monologues en el Día-V de Newfoundland (Canadá), de hablar en voz alta, de ser visibles y de deshacerse del secretismo y la vergüenza ${ }^{9}$. Por esta labor Ensler ha sido nombrada una de las 25 líderes más importantes de su país por el US News Media Group (2009), se la ha invitado a dar testimonio ante el Senado estadounidense sobre las violaciones masivas de mujeres y niñas en la República Democrática del Congo (2009) y ha sido galardonada con el Premio Tony 'Isabelle Stevenson' por su dedicación a causas humanitarias $(2011)^{10}$. En el momento de escribirse estas líneas la autora está pasando por un tratamiento contra el cáncer con efectos más que evidentes ${ }^{11}$, pero continúa siendo la cara y la voz más conocida de un movimiento que, más allá de The Vagina Monologues, sigue generando obras y montajes alrededor del tema de la(s) violencia(s) ejercida(s) contra los cuerpos femeninos y que tiene puntos de contacto con el Teatro de los Oprimidos del dramaturgo brasileño Augusto Boal, como se ha apuntado ya y se explorará con más detalle a continuación. En su

\footnotetext{
${ }^{8}$ KATTWINKEL, Susan, "Spreading American Feminism. The Vagina Monologues and Cultural Identity", en MAUFORT, Marc y DE WAGTER, Caroline (eds.), Signatures of the Past. Cultural Memory in Contemporary Anglophone North American Drama, Bruselas: Peter Lang, 2008, págs. 249-257.

${ }^{9}$ BEAUSOLEIL, Natalie, "Speaking the Unspeakable: An Interview with the Producers of The Vagina Monologues in St. John's, Newfoundland", en Atlantis, vol. 27, ${ }^{\circ}$ 2, 2003, <http://forms.msvu.ca/atlantis/vol/272all/272beausoleil.pdf>. Fecha de acceso: $14 \mathrm{de}$ marzo de 2011.

${ }_{10}^{10}<w w w . v d a y . o r g>$. Fecha de acceso: 19 de abril de 2011.

${ }^{11}$ Véanse, por ejemplo, las siguientes imágenes: <http://healthbistro.lifescript.com/wpcontent/uploads/2010/10/EveEnsler102610.jpg>

$<$ http://cache.daylife.com/imageserve/OfROgAvffLccG/439x.jpg>; $<$ http://cancernaturalremedies.com/wp-content/uploads/2011/02/Eve-Ensler.jpg>.

Futhark 7 (2012) Fernández, Más allá de los cuerpos dóciles, 131-161
}

ISSN $1886-9300$ 
contribución al Boal Companion de 2006 Suzanne Lacy hablaba del terreno común que ella identificaba entre el trabajo de Boal y el performance art feminista de los Estados Unidos ${ }^{12}$. Yo considero que la producción dramática de Ensler, a pesar de no estar catalogada dentro del performance art propiamente dicho, puede localizarse también en ese campo compartido. En él, el teatro se convierte en una forma de hacer política (entendida ésta en un sentido amplio, como todo aquello relacionado con la res publica, más allá del reduccionismo partidista) en la que los roles tradicionales se cuestionan y el público espectador puede pasar a ser actor, diluyendo las tradicionales dicotomías 'productor/receptor' y 'activo/pasivo'. En esa intersección entre dos formas contemporáneas de entender la puesta en escena (drama boaliano y teatro feminista) se cumple el principio ya clásico del movimiento de liberación de las mujeres: lo personal es político.

Como explica Ann E. Armstrong en un artículo también incluido en el Boal Companion, el movimiento feminista -al que el trabajo de Eve Ensler se adscribe sin ninguna duda- y el Teatro de los Oprimidos comparten la finalidad de despertar conciencias. Es el proceso llamado en inglés consciousness-raising, que fue fundamental en el desarrollo de la que en el mundo anglófono se llama Segunda Ola del Feminismo -la que surgió a finales de los años sesenta del siglo $\mathrm{XX}$ - y que Ensler reconoce, como se recoge en las primeras líneas de este artículo, como su misión fundamental. Un proceso de transformación que pasa, tal cual detalla la propia Armstrong ${ }^{13}$, por varias etapas de reflexión y pensamiento crítico y que, idealmente, debería culminar en algún tipo de acción enfocada hacia el cambio. En Theatre of the Oppressed Boal dejaba bien claro que "cambio" era precisamente la palabra clave de su propuesta dramatúrgica: "Theater is change and not simple presentation of what exists: it is becoming and not being"14. Aquí el espectador

\footnotetext{
${ }^{12}$ LACY, Suzanne, "Activism in Feminist Performance Art", en COHEN-CrUZ, Jan; SchutZman, Mady (eds.), A Boal Companion. Dialogues on Theatre and Cultural Politics, Nueva York y Londres, Routledge, 2006, pág. 91.

${ }_{13}$ ARMSTRONG, Ann Elizabeth, "Negotiating Feminist Identities and Theatre of the Oppressed", en COHEN-CruZ, Jan; SchutZMAN, Mady (eds.), A Boal Companion. Dialogues on Theatre and Cultural Politics, Nueva York y Londres, Routledge, 2006, pág. 178.

${ }^{14}$ BOAL, Augusto, Theatre of the Oppressed, Nueva York, Theatre Communications Group, 1985 (1974), pág. 28.

Futhark 7 (2012) $\quad$ Fernández, Más allá de los cuerpos dóciles, 131-161
}

ISSN $1886-9300$ 
adquiere una nueva función, las tramas al uso pueden reinventarse, el espacio escénico se redefine... el teatro se convierte así en una plataforma de acción y, si no en una revolución de facto, al menos sí en el ensayo para la revolución ${ }^{15}$. En su concepción del teatro por la igualdad, Ensler habla de ligar la intención transformadora con el proceso de hacer arte ${ }^{16}$. En su marco del drama para la liberación de los grupos oprimidos, Boal explica cómo su propuesta permite imaginar alternativas a los conflictos y extrapolar lo visto en escena a la realidad cotidiana ${ }^{17}$. En ambos casos forma y contenido van ligados; no hay telón que separe el teatro de la vida.

\section{El legado de Augusto Boal y su adaptación 'ensleriana'}

"Nada es ajeno a la política."

(Augusto Boal)

El portal de Internet <www.vday.org $>$ hace una clara llamada a la acción: "Tú puedes ayudar a acabar con la violencia contra las mujeres y las niñas en tu comunidad", asegura, "pasos sencillos y concretos pueden cambiar el mundo" ${ }^{18}$. El punto de partida de ese cambio, como se ha venido comentando, es el teatro: producciones de algunos textos de Eve Ensler, que renuncia a los derechos de autora durante un mes al año, previo acuerdo con las organizaciones que se comprometen a organizar los eventos del Día-V. La puesta en escena actúa como catalizador para la transformación social, y el público forma parte fundamental del proceso. En este sentido, el proyecto parece beber de las enseñanzas de Augusto Boal, para quien el teatro era un arma eficaz; tanto que las clases dominantes siempre han tratado de controlarlo y usarlo como herramienta para la dominación ${ }^{19}$. Con su Poética de los Oprimidos Boal perseguía un objetivo muy específico: la liberación del público espectador, que a través de ejercicios como el Teatro Fórum, el Teatro Invisible, etc., podía pasar a la acción primero en el entorno controlado de la puesta en escena y después (idealmente) en la vida real. Los

\footnotetext{
${ }^{15}$ BOAL 1985: 122.

${ }^{16}$ GREENE 2001: 164.

${ }^{17}$ BOAL 2006a: 40.

${ }^{18}<$ www.vday.org/take-action>; mi traducción. Fecha de acceso: 19 de abril de 2011.

${ }^{19}$ BOAL 1985: ix:

Futhark 7 (2012) Fernández, Más allá de los cuerpos dóciles, 131-161
}

ISSN $1886-9300$ 
espectadores se convertían así en "espect-actores"; en agentes de cambio a través de la acción:

[T] he poetics of the oppressed focuses on the action itself: the spectator delegates no power to the character (or actor) either to act or to think in his place; on the contrary, he himself assumes the protagonic role, changes the dramatic action, tries out solutions, discusses plans for change -in short, trains himself for real action. [...] The liberated spectator, as a whole person, launches into action. No matter that the action is fictional; what matters is that it is action! ${ }^{20}$

En el caso de formas 'boalianas' concretas como el Teatro Fórum, por ejemplo, la transformación del espectador en actor es literal, en el sentido de que quien está viendo una obra tiene la posibilidad de congelar la acción y subir al escenario para ocupar el sitio del protagonista. El intérprete profesional es reemplazado así por un miembro de la audiencia a quien se le ocurre una forma alternativa de continuar la trama, dando salida a un conflicto con el que se identifica de alguna manera. La idea es que los textos presenten situaciones de injusticia o abuso de poder reconocibles para quien asiste al espectáculo, y que el público pueda ensayar en sus propias carnes y por propia iniciativa cómo luchar contra ellas dentro del espacio estético previamente creado. Como explica Boal en The Aesthetics of the Oppressed, la espectadora (él utiliza sistemáticamente el pronombre femenino "she" cuando habla de "spectator" en sus últimos libros), al invadir la escena, practica de forma consciente un acto de responsabilidad: el escenario es una representación de lo real; sin embargo, la espectadora no es ficticia: existe en la escena y más allá. Entrando en la ficción del teatro, la espectadora actúa y practica, no sólo en esa ficción, sino también en la realidad social a la que pertenece. Está simultáneamente en dos mundos $^{21}$, y pasa por un proceso de transformación ya sólo con su mera participación. Al fin y al cabo, Boal entiende que la preparación es en sí misma una forma de acción ${ }^{22}$, y que pensar y reflexionar

\footnotetext{
${ }^{20}$ BOAL 1985: 122.

${ }^{21}$ BOAL, Augusto, The Aesthetics of the Oppressed, Londres y Nueva York, Routledge, 2006b, pág. 74.

22 "[H]ere to be a spectator means to prepare oneself for action, and preparing oneself is already in itself an action" (BOAL 2006a: 72).
}

Futhark 7 (2012) Fernández, Más allá de los cuerpos dóciles, 131-161

ISSN $1886-9300$ 
también son formas de actuar $^{23}$; de ahí la importancia de la toma de conciencia de la que venimos hablando y que tan relevante es también para Eve Ensler.Un ejemplo de temática centrada en el género y el cuerpo femenino sobre la que tanto Boal como Ensler han trabajado en su labor en favor de la igualdad sería, por ejemplo, la abordada en el montaje de Teatro Fórum A Maré da Vida, dirigido por Geo Britto, del laboratorio de Teatro de los Oprimidos de Río de Janeiro, y recogido en imágenes en el volumen The Aesthetics of the Oppressed. Como una parte importante del drama de Eve Ensler, este ejercicio de participación activa de los espectadores/as se centra en la violencia contra las mujeres, presentando situaciones de agresión que no resultan extrañas al público, pero que han estado silenciadas como "problemas domésticos" durante demasiado tiempo. En una línea similar, la obra Periferia e Ação, dirigida por Roni Valk e incluida por Boal en el mismo libro de 2006, tiene como tema principal el embarazo adolescente, uno de los asuntos a los que dedica una sección la pieza de Ensler I Am an Emotional Creature. Donde el brasileño sube a las tablas a chicos y chicas jóvenes que tratan la cuestión dialogando desde sus propias perspectivas, la estadounidense incluye datos estadísticos de su país en forma de "Girl Facts" objetivos, que a su vez abren la puerta a dos monólogos sobre las relaciones sexuales en la adolescencia. En el primero la protagonista, embarazada sin quererlo, se encuentra en una encrucijada entre las lecciones de moral que le dan la sociedad y su familia y su propio deseo de posponer la maternidad hasta que esté preparada. El segundo se centra en la pregunta "¿qué es una buena chica?", y combina varias respuestas a partir de entrevistas con jóvenes reales:

\section{"It's Not a Baby, It's a Maybe"}

[...] I knew I wasn't supposed to be doing it I was practising abstinence apply it. but to be honest, I didn't really know how to

'Cause once the kissing starts...

I am tired a lot.

My mother thinks I'm doing drugs.

\footnotetext{
23 "[T]hinking is a form of action" (BOAL 2005: 128).

Futhark 7 (2012) Fernández, Más allá de los cuerpos dóciles, 131-161
}

ISSN 1886-9300 
I could never tell her.

She is super-Catholic.

[...] I wouldn't be attacking it or anything.

I would just be removing it.

I wouldn't hurt it,

just put it someplace else.

It is not a person really.

It's a problem

getting bigger and bigger ${ }^{24}$.

\section{“What's a Good Girl?"}

She doesn't speak to a boy at all

Has morals

Tells the truth even if she pisses people off

Respectable

Doesn't argue

Polite

Quiet

She brings her homework with her

Doesn't step out of the line

Follows her parents in everything

Goes to church every Sunday

Stays in on the weekends

Doesn't know more than she should

answers $^{25}$.

Asks questions even I she knows the

Algunas de las ramificaciones del marco teórico-práctico de Boal llevan la capacidad de actuar de los espectadores/as mucho más allá del escenario y de su propio ámbito de influencia individual cotidiana, produciendo un impacto directo en toda la comunidad. Así, por ejemplo, el Teatro Legislativo logró elaborar colectivamente y llevar a debate en el Ayuntamiento de Río de Janeiro varias leyes y directivas municipales, de las cuales algunas fueron de hecho aprobadas y puestas en marcha. La Ley 1023/95 de Cuidado

${ }^{24}$ EnSLER, Eve, I Am an Emotional Creature. The Secret Life of Girls around the World, Nueva York: Villard, 2010, págs. 23 y 24.

${ }^{25}$ ENSLER 2010: 27.

Futhark 7 (2012)

Fernández, Más allá de los cuerpos dóciles, 131-161

ISSN 1886-9300 
Geriátrico, por ejemplo, obligaba a todos los hospitales de la ciudad a ofrecer tratamiento especializado a las personas mayores, y había sido concebida por el grupo Terceira Idade de la iniciativa 'boaliana' de Teatro Legislativo; una célula del Teatro de los Oprimidos formada por ciudadanos/as de más de 60 años ${ }^{26}$. Aquí los "espectactores", con la mediación de Boal como concejal y su equipo de jokers ${ }^{27}$, llevaron sus ensayos a buen puerto, y la praxis con los colectivos oprimidos pasó de cultivar un teatro político a, literalmente, hacer política a partir del ejercicio dramático:

Our mandate's project is to bring theatre back into the centre of political action -the centre of decisions- by making theatre as politics rather than merely making political theatre. In the latter case, the theatre makes comments on politics; in the former, the theatre is, in itself, one of the ways in which political activity can be conducted ${ }^{28}$.

Esta forma de entender el término "actor" no sólo como intérprete dentro del espacio estético, sino como agente en la sociedad que le rodea, es la que vincula más directamente el Teatro de los Oprimidos con la dramaturgia feminista de Eve Ensler. Centrada como decíamos en la experiencia de las mujeres y las niñas, no ha generado por ahora directivas concretas como el Teatro Legislativo de Boal, pero sí ha llamado la atención de las instituciones políticas -recordemos el mencionado testimonio de Ensler sobre las violaciones en Congo en el Senado de EE.UU- y ha movilizado a miles de personas dentro y fuera de la iniciativa Día-V.

En primera instancia y como forma más evidente de tomar parte en el hecho teatral, en las puestas en escena de las obras de Ensler el público es invitado a ser activo, ya sea cantando a coro en voz bien alta palabras tabú, como en el monólogo "Reclaiming cunt" de The Vagina Monologues ("Reivindicar el coño" -hay que tener en cuenta que cunt es la peor palabra de la lengua inglesa, y que

\footnotetext{
${ }^{26}$ BOAL 2005: 95.

${ }^{27}$ En el Teatro de los Oprimidos en general, los/as jokers son maestros/as de ceremonias; facilitadores/as del proceso participativo en la puesta en escena. En el Teatro Legislativo en particular también actúan como enlace entre los distintos grupos que montan los espectáculos comunitarios y quienes les representan como legisladores (en el caso del Ayuntamiento de Río en los años 90, el propio Augusto Boal).

${ }^{28}$ BOAL 2005: 20.
}

Futhark 7 (2012)

Fernández, Más allá de los cuerpos dóciles, 131-161

ISSN $1886-9300$ 
gritarla es muy poco habitual para las estadounidenses); ya sea participando en alguna actividad paralela de toma de conciencia en el entreacto. Esto ocurrió por ejemplo en el Día-V 2006 de Providence, en Rhode Island (EE.UU.), donde se subastó una "Barbie Presidenta" que potenciaba la imagen de las mujeres como figuras empoderadas en el mundo de la política. Así, dentro del marco del Día-V la obra dramática se entiende como parte de un evento teatralizado más amplio, y no como mera reproducción de un texto literario sobre las tablas. Aunque los trabajos de Ensler suelen tener formato de monólogo, la posibilidad de un diálogo transformador está presente tanto en el texto en sí como en el proceso de recepción. Por un lado hay que tener en cuenta las conversaciones previas de la autora con cientos de mujeres de distintos países, edades y clases sociales (más de 200 sólo para la primera versión de The Vagina Monologues) ${ }^{29}$, que constituyen un diálogo multicultural enmarcado dentro del proceso creativo de las piezas y que produce efectos directos en las entrevistadas. La anciana que habla de la "inundación" que sufrió tras su primer beso en The Vagina Monologues, por ejemplo, confiesa al final de su intervención: "You know, actually, you're the first person I ever talked to about this, and I feel a little better" ${ }^{30}$. Por otro lado, cabe recordar aquí la función de conversación pública que la propia Ensler concibe para su teatro: "As I perform them [the monologues], I am able to create a platform for dialogue among women"31. Sus obras, por lo tanto, a pesar de ser en su mayoría monologadas, conservan el elemento movilizador que Augusto Boal ha reconocido en otros textos no dialogados puestos en escena por mujeres y que para él mantienen intactos su teatralidad y su potencial político:

$[T]$ he smallest theatrical unity is two people. And now someone will say: 'What about monologues?'

Once I saw a production of a very beautiful play, which told the story of a woman from the time she got home till the moment of her suicide: the theatricality was engendered by the intense and extreme interrelation between the woman and the phone which didn't ring, the doorbell which didn't sound, the friend or

\footnotetext{
${ }^{29}$ ENSLER, Eve, The Vagina Monologues, Nueva York: Villard, 1998, pág. 4.

${ }^{30}$ ENSLER 1998: 30.

${ }^{31}$ GREENE 2001: 164.

Futhark 7 (2012)

Fernández, Más allá de los cuerpos dóciles, 131-161
}

ISSN 1886-9300 
parent or lover who didn't call on her -the interrelation between

the woman herself and the powerful presence of absence ${ }^{32}$.

Mediante la puesta en escena a través de este tipo de monólogos sobre experiencias diversas de violencias e injusticias determinadas por el género y ejercidas en su mayoría sobre el cuerpo femenino, el teatro de Eve Ensler pretende generar en todas las personas implicadas la necesidad de seguir actuando más allá de la preparación y la representación de las piezas dramáticas. No se trata de repetir sobre el escenario una colección de anécdotas inconexas sin sentido global y por puro entretenimiento, sino de abrir el ángulo sobre los temas tratados, pasando de la experiencia individual a la colectiva y buscando los puntos en común entre las mujeres entrevistadas previamente a la elaboración del texto, la autora/performer durante el proceso creativo y de escenificación inicial, las actrices que continúan el proyecto como profesionales y/o dentro del marco del Día-V y las espectadoras que se acercan a él para participar de las obras como productos artísticos y/o políticos. Igualmente, en la praxis del Teatro de los Oprimidos de Boal se parte de una situación concreta propuesta por una persona particular para llegar a debatir sobre la desigualdad en términos de grupo. Es función de los/as jokers demostrar que no hay ningún problema que sea exclusivo de un solo individuo. Este maestro/a de ceremonias es quien debe encontrar los elementos de injusticia compartidos en distintos momentos cotidianos para convertir el ejercicio dramático en un proceso significativo para todas las personas participantes. Como explica el creador de esta iniciativa, no debe minusvalorarse la impresión de cada uno/a sobre la propia opresión, pero los problemas deben poder pluralizarse ${ }^{33}$. Es lo que se llama en la terminología del Teatro de los Oprimidos "ascesis"; el movimiento del fenómeno puntual a la ley general que lo explica: "[W]e work on the case of an individual, and from that individual case we extrapolate into the group present, and then, sometimes, from that group into the larger society of which it is a microcosm or a fragment" ${ }^{34}$.

Según explica Sal Renshaw en relación con The Vagina Monologues -con un argumentario que hago extensivo a piezas de

\footnotetext{
${ }^{32}$ BOAL 2005: 49

${ }^{33}$ BOAL 2005: 47.

${ }^{34}$ BOAL 2006a: $x x$.

Futhark 7 (2012)

Fernández, Más allá de los cuerpos dóciles, 131-161
}

ISSN $1886-9300$ 
Ensler más recientes y de factura similar- las mujeres implicadas en el teatro de la norteamericana se narran y se entienden a sí mismas a través de sus experiencias inter-subjetivas, descubriendo que las otras son parte ineludible de su 'yo', ${ }^{\prime 3}$. Con sus obras polifónicas, pues, y según explica la propia Ensler refiriéndose concretamente a The Vagina Monologues, se promueve la unidad y se rompe el aislamiento de las mujeres ${ }^{36}$. El significado último de la experiencia viene dado no sólo por la recepción de cada individua de forma independiente, sino por la identificación grupal con lo visto. Sin duda alguna, como arguye Kattwinkel en relación con su obra más conocida, las obras de Ensler no retratan todos y cada uno de los elementos culturales vinculados a cada mujer de cada país cuyo testimonio se incluye, simplificando el retrato en aras de un reconocimiento mutuo global. Esto es así porque la autora aboga por buscar lo que todas las mujeres tenemos en común, siendo la amenaza de la violencia (física, psicológica, simbólica, económica) el hilo conductor de sus textos de la última década y probablemente nuestro rasgo más ampliamente compartido, como se apunta en The Vagina Monologues, The Good Body, Fur Is Back y I Am an Emotional Creature, respectivamente:

[B]ad things are happening to vaginas everywhere: 500,000 women are raped every year in the United States; 100 million women have been genitally mutilated worldwide; and the list goes on and on ${ }^{37}$.

I have been to more than forty countries in the last six years. I have seen the rampant and insidious poisoning: skin-lightening creams sell as fast as tooth-paste in Africa and Asia; the mothers of eight-year-olds in America remove their daughters' ribs so they will not have to worry about dieting; five-year-olds in Manhattan do strict asanas so they won't embarrass their parents in public by being chubby; girls vomit and starve

\footnotetext{
35 Renshaw, Sal, "Divine Gifts and Embodied Subjectivities in The Vagina Monologues", en International Feminist Journal of Politics, 6.2, junio 2004, pág. 318. ${ }^{36}$ GREENE 2001: 164.

${ }^{37}$ ENSLER 1998: xxii.

Futhark 7 (2012)

Fernández, Más allá de los cuerpos dóciles, 131-161
}

ISSN 1886-9300 
themselves in China and Fiji and everywhere; Korean women remove Asia from their eyelids... the list goes on and on ${ }^{38}$.

What's up? What's fucking up? Don't you read the news? The Amish girls shot down in the school 'cause they were there, 'cause they were girls. Or the girls in the refugee camp in Darfur going to get grass for their donkeys or wood for the fire who get grabbed, who get raped and raped and can't find their way back. [...] Women are dying. Women have their labia ripped off in the Congo, their faces melted off in Pakistan, they are bought as children in Atlanta ${ }^{39}$.

Everyone seems to have a certain way they want you [girls] to be -your mother, father, teachers, religious leaders, politicians, boyfriends, fashion gurus, celebrities, girlfriends. In researching this book I came upon a very disturbing statistic: 74 percent of you say that you are under pressure to please everyone. [...] To please the fashion setters, we starve ourselves. To please boys, we push ourselves when we aren't ready. To please the popular girls, we end up acting mean to our best friends. To please our parents, we become insane overachievers ${ }^{40}$.

Sin embargo, la propia Kattwinkel reconoce, a pesar de sus críticas a la relativa ceguera cultural de Ensler, y de nuevo hablando de The Vagina Monologues, que aun sin las especificidades no contempladas, ciudadanas de todo el mundo han encontrado inspiración en los espectáculos de esta autora y han usado sus textos como herramienta para abrir discusiones válidas sobre sus propias comunidades ${ }^{41}$. Bell y Reverby, investigadoras en temas de género y activistas del movimiento feminista, hacen una lectura parecida de la pieza (extensible al resto de la producción de Ensler): aunque, por ejemplo, en el texto se minimicen las diferencias entre las mujeres africanas 0 las de países muy fragmentados culturalmente como Bosnia o Afganistán, las entrevistas con personas que han participado en montajes dentro del Día-V ponen de manifiesto que las puestas en escena tienen un poder de

\footnotetext{
${ }^{38}$ ENSLER, Eve, The Good Body, Londres, William Heinemann, 2001, pág. xiii.

${ }^{39}$ EnSLER, Eve, Fur Is Back, en EnSLER, Eve (ed.), A Memory, A Monologue, A Rant, and a Prayer. Writings to Stop Violence against Women and Girls, Nueva York, Villard, 2007, págs. 177 y 178.

${ }^{40}$ ENSLER 2010: xxiii y xxiv.

${ }^{41}$ KATTWINKEL 2008: 252.

Futhark 7 (2012) Fernández, Más allá de los cuerpos dóciles, 131-161
}

ISSN $1886-9300$ 
concienciación y movilización que se mantiene en el tiempo ${ }^{42}$. Ejemplo de ello también son los miles de eventos del Día-V 2011 en todo el globo que se recogen en el mapa de la fundación en $<$ http://events.vday.org/> y las más de 900 jóvenes registradas como activistas de la rama Chicas-V en <http://v-girls.ning.com>. Vemos, por lo tanto, cómo además de lo que sucede durante la mise en scène, y en la línea del Teatro de los Oprimidos, que pretende que la comunidad participe directamente de los procesos que le afectan, el teatro de Ensler genera compromiso social y activismo compartido entre muchas mujeres (y no pocos hombres) de distintas generaciones, consiguiendo, en términos sociológicos, que sus obras tengan resonancia más allá de los eventos teatrales propiamente dichos. Entendiendo el término "actor" como sinónimo de "agente consciente de cambio", podemos afirmar entonces que una parte de su público da el paso 'boaliano' de espectador a "espect-actor", como demuestra la tendencia creciente del Día-V. El proyecto dramático y dramatúrgico de Ensler, en pocas palabras, mueve a la acción tanto como el de Boal, si bien cada uno lo hace por sus propios medios y con estrategias particulares.

\section{Cuerpo(s) femenino(s) y empoderamiento: más allá de la docilidad}

Además del objetivo claro de movilización sociopolítica, los dos autores que tratamos aquí comparten el conocimiento y la consciencia sobre la importancia del cuerpo en los procesos que ponen en marcha. Para Augusto Boal el cuerpo humano era la primera palabra del vocabulario teatral, y la transformación de espectador a "espect-actor" pasaba por los siguientes estadios:

1) Conocer el propio cuerpo, sus limitaciones y posibilidades, sus distorsiones sociales y sus capacidades de rehabilitación.

\footnotetext{
${ }^{42}$ BeLL, Susan E.; Reverby, Susan M, "Vaginal Politics: Tensions and Possibilities in The Vagina Monologues", en Women's Studies International Forum, 28, 2005, págs. 440 y 441.
}

Futhark 7 (2012)

Fernández, Más allá de los cuerpos dóciles, 131-161

ISSN $1886-9300$ 
2) Dotarlo de expresividad para buscar las formas de comunicar el propio 'yo' e ir más allá de las formas de expresión habituales.

3) Construir un lenguaje teatral vivo y presente a partir del cuerpo.

4) Tomar conciencia del valor del teatro como discurso para trabajar ciertos temas o ensayar ciertas acciones a partir del cuerpo del espectador-cum-actor ${ }^{43}$.

El trabajo del dramaturgo brasileño, por lo tanto, iba mucho más allá de la palabra escrita de la literatura dramática tradicional para convertirse, mediante el uso del cuerpo explícito dentro del espacio estético, en un acto 'performativo', con todo el poder de transformación que autoras como Erika Fischer-Lichte reconocen a estas prácticas. Según esta crítica alemana, en este tipo de puestas en escena que diluyen los límites tradicionales entre teatro y vida, representación y realidad, el público se sitúa en un espacio liminal que le permite moverse a un tiempo dentro y fuera de los órdenes y normas prescritas, cuestionando así las barreras artificiales que determinan que "esto es teatro" mientras que "esto es una situación social o política" ${ }^{44}$.

En el teatro de Eve Ensler que se analiza en este trabajo el cuerpo (femenino) es tema, materia y vehículo de comunicación. Mediante sus escenificaciones de monólogos construidos a partir de entrevistas con mujeres de carne y hueso, el trabajo de esta autora trata, en sus propias palabras, de hacer avanzar la relación de las mujeres con sus cuerpos ${ }^{45}$, intentando que las participantes en el hecho teatral superen sus miedos, prejuicios y tabúes para conseguir empoderarse precisamente a partir de aquello que el patriarcado ha secuestrado y maltratado durante siglos. Como afirma Gloria Steinem en su prólogo a The Vagina Monologues, el valor de esta pieza ( $y$, añadiría yo, de otras de la misma autora que comparten su temática y estrategias) es que va "beyond purging a past full of negative attitudes. It offers a personal, grounded-in-the-

\footnotetext{
${ }^{43}$ Adaptado de BOAL 1985: 125 y 126.

44 FISCHER-LICHTE, Erika, The Transformative Power of Performance. A New Aesthetics, Londres y Nueva York, Routledge, 2008, pág. 177.

${ }^{45}$ GREENE 2001: 167.

Futhark 7 (2012) Fernández, Más allá de los cuerpos dóciles, 131-161
}

ISSN 1886-9300 
body way of moving toward the future" ${ }^{46}$. Se trata de ir más allá de la queja y el lamento, superando la condición de víctima y construyendo un espacio de autoconocimiento, solidaridad y recuperación en el que el cuerpo de las mujeres se rebele contra las discriminaciones explícitas o implícitas de su cultura y sociedad. Las piezas de Ensler tratan, por lo tanto, de identificar primero los mecanismos de opresión forzados desde fuera o autoimpuestos por las propias mujeres para luego denunciarlos y, en última instancia, romper con ellos para seguir avanzando hacia la igualdad real. Así, por ejemplo, en The Good Body se encarnan sobre el escenario las experiencias de mujeres de diferentes países y edades con sus cuerpos jóvenes o viejos, flacos u obesos, operados o naturales, expuestos 0 escondidos. Mediante la dramatización de circunstancias dispares que confluyen en el cuerpo de la propia Ensler $\mathrm{u}$ otras actrices sobre el escenario se exploran los mecanismos de lo que Pierre Bourdieu ha llamado "violencia simbólica", que consigue convertir a las mujeres en víctimas y perpetradoras a un tiempo. Este término hace referencia en el marco del pensamiento del sociólogo francés a la forma de violencia que se ejerce sobre un agente social con su complicidad ${ }^{47}$, y se presenta como una noción "indispensable para explicar fenómenos aparentemente tan diferentes como la dominación personal en sociedades tradicionales o la dominación de clase en las sociedades avanzadas, las relaciones de dominación entre naciones (como el imperialismo o el colonialismo) o la dominación masculina tanto en las sociedades primitivas como modernas" ${ }^{48}$.

El último punto mencionado por Fernández es el que se hace más evidente en la producción dramática de Eve Ensler, centrada casi al cien por cien en cuestiones de género que, si bien interactúan con otras variables como la clase, la edad, la nacionalidad o la identidad sexual, se ponen en primer plano como problemáticas transversales y de carácter global, como se ha venido

\footnotetext{
${ }^{46}$ Steinem, Gloria, "Foreword", en EnSLER, Eve, The Vagina Monologues, Nueva York: Villard, 1998, pág. xiv (énfasis añadido).

47 BouRDIEU, Pierre; WACQUANT, Loïe, Réponses. Pour une anthropologie réflexive, Paris: Seuil, 1992, pág. 142.

${ }^{48}$ Fernandez, J. Manuel, "La noción de violencia simbólica en la obra de Pierre Bourdieu: una aproximación crítica", en Cuadernos de Trabajo Social, 18, 2005, pág. 8 (énfasis añadido).
}

Futhark 7 (2012)

Fernández, Más allá de los cuerpos dóciles, 131-161

ISSN $1886-9300$ 
apuntando. En sus textos los procesos de socialización femenina a través de la violencia simbólica adquieren una gran relevancia, ya que se muestran los resultados de estrategias discriminatorias que en ocasiones logran convertir a las mujeres en partícipes de su propia opresión, en la línea de lo propuesto por Bourdieu. El colectivo discriminado va asumiendo los valores y aceptando como correctas las ideas del grupo dominante, hasta el punto de ejercer violencia sobre su propio cuerpo para adaptarse a las convenciones que ha interiorizado como las únicas válidas. Es un fenómeno similar a lo que otro pensador francés, Michel Foucault, llamó el "sujeto auto-regulado" producido por los mecanismos de biopoder: aquél que ha sido moldeado y aleccionado por el sistema (a menudo a base de dinámicas de premio-castigo más o menos evidentes) y ha acabado por asumir la responsabilidad de controlarse a sí mismo para no salirse de las normas ${ }^{49}$. Es, al fin y el cabo, lo que Augusto Boal llama en su teoría del Teatro de los Oprimidos el "policía en la cabeza" (Cop in the Head), que ejerce de autocensor de pensamientos y acciones, vigilante de los establecido y garante de las estructuras de dominación aun cuando el individuo no sea consciente de ello:

\begin{abstract}
We define as 'cop' the image present in our heads, at a point of action, which obliges us to do what we don't want to do or prevents us from doing what we want to do. Its presence means that our desire is diluted and that, instead of enacting our own desire, we enact the 'cop's'. This can come about by violence or by seduction, by toughness or gentleness, by word or by gesture, with audacity or timidity ${ }^{50}$.
\end{abstract}

Una buena ilustración de esta idea compartida por los tres teóricos y escenificada por Ensler nos la proporciona en The Good Body la sección dedicada a Tiffany, una modelo de treinta y cinco años que se somete voluntariamente $(i$ ?) a la obsesión de su marido, cirujano plástico, por alcanzar la perfección -la de ella. Como hiciera la sumisa Georgiana del cuento decimonónico "The

\footnotetext{
49 DANAHER, Geoff; SchiRATO, Tony; WeBB, Jen, Understanding Foucault, Londres: Sage, 2000, pág. 75.

${ }^{50}$ BOAL 2006a: 149.

Futhark 7 (2012)

Fernández, Más allá de los cuerpos dóciles, 131-161
}

ISSN $1886-9300$ 
Birthmark" ${ }^{51}$, Tiffany se pone en manos de su amo, el científico todopoderoso, para que la moldee a su gusto, hasta el punto de acabar reconociéndose como una creación suya ${ }^{52}$. Igualmente, Carol, una cuarentona judía cuya voz también destila Ensler en esta obra sobre el cuerpo, lee en Cosmopolitan sobre la cirugía para rejuvenecer la vagina y decide someterse a ella para hacer feliz y volver a excitar sexualmente a su compañero. El monólogo, sin embargo, no esconde las consecuencias de la intervención: Harry está encantado de poder volver a penetrar un órgano sexual que parece el de una jovencita, pero Carol reconoce que siente muchísimo dolor, que ha llegado a llorar durante el acto y que a veces desearía poder pararlo todo y poner un cartel de "cerrado" como si su vagina fuera una tienda ${ }^{53}$.

En una línea similar, pero elaborando el texto a partir de testimonios de mujeres mucho más jóvenes, I Am an Emotional Creature también incluye reflexiones sobre lo que las chicas de diferentes países hacen o dejan hacer sobre sus propios cuerpos para conseguir aceptación, autoestima y reconocimiento. Una muchacha estadounidense con trastornos de alimentación confiesa que la presión de su familia le ha hecho controlar su peso desde que estaba en séptimo curso, y que ha acabado automutilándose como estrategia de control de sus emociones ${ }^{54}$. Una adolescente iraní, por su parte, explica que por su decimosexto cumpleaños sus padres le regalaron una operación de nariz para que estuviera más guapa. La pérdida de su apéndice grande, diferente y especial le provoca una gran tristeza, porque ha dejado de reconocerse a sí misma y ha pasado a ser una belleza clonada más:

Pretty girls don't look like anything particular. They look like everyone dreams of looking, but they do not look like anything

51 "The Birthmark" (1843), escrito por el estadounidense Nathaniel Hawthorne, cuenta
la historia de un científico, Aylmer, obsesionado con hacer perfecta a su esposa
Georgiana, cuyo único defecto es una marca de nacimiento en la mejilla. Tras varios
experimentos Aylmer consigue elaborar una poción que borrará la marca, pero al
someter a Georgiana a la operación para hacerlo y atisbar la felicidad al ver
desaparecer por un instante la pequeña mancha, deberá enfrentarse a la muerte de
ella y a su propia culpa por haber querido vencer a la naturaleza y jugar a ser un dios.
52 ENSLER 2001: 37 .
53 ENSLER 2001: 52 .
54 ENSLER 2010: 19.
Futhark 7 (2012) Fernández, Más allá de los cuerpos dóciles, 131-161

ISSN $1886-9300$ 
you can really identify. [...] It is hard to describe, but my nose gave me permission. It inspired me with wicked ideas. It made me daring. [...] My parents who loved me planned, strategized, and eventually succeeded at killing my nose. Murdering it.

On my sixteenth birthday they paid a man to take my nose out. They hired a hit man to take my poor nose down. The only problem is that my nose was attached to $\mathrm{me}^{55}$.

Vemos así cómo la labor de Ensler constituye un ejercicio de denuncia de la violencia simbólica definida por Bourdieu y de visibilización del Cop in the Head 'boaliano' que las mujeres aprendemos a llevar dentro -igual que el resto de la ciudadanía, pero en casos como éstos con consecuencias claramente determinadas por el género. Además, su teatro contiene ecos del pensamiento de Michel Foucault como parte de un proyecto feminista en el que Diamond y Quinby reconocen cuatro puntos de convergencia con el pensamiento de este filósofo francés:

1) La identificación del cuerpo como locus de dominación a través del cual se alcanza la docilidad pero también se construye la subjetividad.

2) El énfasis en las operaciones íntimas y locales del poder (los micropoderes por debajo del gran poder del estado).

3) La focalización en el papel que el discurso juega en la producción y el sostenimiento del poder.

4) La crítica a los grandes universales sobre la verdad, la libertad y la naturaleza humana ${ }^{56}$.

En este sentido, el drama de esta autora, como hemos visto en los ejemplos anteriores, critica los mecanismos de violencia que el patriarcado ejerce u obliga a ejercer sobre los cuerpos femeninos, pero también y sobre todo, asume la idea 'foucaultiana' tardía de que donde hay poder existe la posibilidad de resistencia. Como recuerda Lois McNay, tras su articulación de las teorías sobre la sujeción de los ciudadanos/as en sus primeras obras, Foucault planteó la

\footnotetext{
${ }^{55}$ ENSLER 2010: 64 y 65.

${ }^{56}$ DIAMOND, Irene; QUINBY, Lee, "Introduction”, en DIAMOND, Irene; QUINBY, Lee (eds.), Feminism \& Foucault. Reflections on Resistance, Boston, Northeastern University Press, 1988, pág. $x$.

Futhark 7 (2012)

Fernández, Más allá de los cuerpos dóciles, 131-161
}

ISSN 1886-9300 
posibilidad de que los individuos fueran más allá de ser cuerpos dóciles y pudieran llegar a ser agentes con la capacidad de retar a las estructuras de dominación de su sociedad: "Individuals are no longer conceived as docile bodies in the grip of an inexorable disciplinary power, but as self-determining agents who are capable of challenging and resisting the structures of domination in modern society" ${ }^{57}$. Pensamiento éste del teórico francés que, de nuevo, coincide con el de Augusto Boal, para quien cada persona oprimida era un subversivo subyugado: "His submission is his Cop in the Head, his introjection. But he also possesses the other element, subversion" ${ }^{58}$. La función del teatro, según el brasileño, era precisamente dinamizar ese potencial para la subversión y neutralizar al Cop in the Head hasta hacerlo desaparecer.

Dando forma a estas ideas a través de los testimonios monologados de distintas mujeres, Ensler muestra en The Good Body, como veíamos, a una serie de personajes esclavos del canon de belleza occidental: a las operadas Tiffany y Carol que mencionábamos antes les acompañan una adolescente obligada por sus padres a pasar sus vacaciones en un campamento para obesas, una anciana de ochenta años que se arrepiente de los "arreglos" que se hizo a lo largo de su vida y un grupo de mujeres indias que, imitando a su reina de la belleza nacional, quien llegó a Miss Mundo, pasan horas en el gimnasio y se matan de hambre para convertirse en "Miss Skinny India World" ${ }^{59}$. Pero, por otra parte, Ensler también presenta como alternativa empoderante una serie de cuerpos rebeldes que toman la palabra: aparece Isabella Rossellini denunciando a la industria cosmética por firmar el acta de defunción simbólica de cualquier top model que pase de los cuarenta: "They sent me so many flowers on my fortieth birthday. I knew I was dead. [...] They fired me because I was strong. They told me not to talk. I am talking. I am talking" ${ }^{60}$. Y vemos a Dana, una artista veinteañera de tatuajes y piercings, defendiendo su derecho a decidir sobre su aspecto y su identidad: "My piercings are about evolving multiple definitions of myself as a woman, as a lesbian. These metal bars

57 MCNAY, Lois, Foucault \& Feminism: Power, Gender and the Self, Boston, Northeastern University Press, 1992, pág. 4.

${ }^{58}$ BOAL 2006a: 42 (énfasis añadido).

${ }^{59}$ ENSLER 2001: 80.

${ }^{60}$ ENSLER 2001: 64 y 65.

Futhark 7 (2012) Fernández, Más allá de los cuerpos dóciles, 131-161

ISSN $1886-9300$ 
break open the way for me to be complicated. No one is going to tell me who the fuck I $\mathrm{am}^{\prime \prime 61}$. Finalmente, terminando la obra en la trastienda de una cafetería en Afganistán donde un grupo de activistas arriesga su vida al transgredir los preceptos talibanes y comerse un helado, Ensler lanza un mensaje que enfatiza la experiencia compartida de las mujeres del mundo a través del "nosotras" y anima a apreciar el propio cuerpo tal cual es: "Our body is our country, the only city, the only village, the only every we will ever know" ${ }^{\prime 2}$. Como ella misma ha escrito en la introducción al texto, la idea clave aquí es la necesidad y posibilidad de reforzar la autoestima femenina para evitar el miedo y la violencia autoimpuesta: "Tell the image makers and magazine sellers and the plastic surgeons that you are not afraid. That what you fear the most is the death of imagination and originality and metaphor and passion. Then be bold and LOVE YOUR BODY. STOP FIXING IT. It was never broken" ${ }^{63}$.

En el universo 'ensleriano' el paso de la docilidad a la rebeldía se pone en escena a través del lenguaje verbal, pero también del no verbal, como es lógico teniendo en cuenta que hablamos de teatro: se da testimonio sobre la posibilidad de subversión mediante el monólogo, como hemos venido viendo con The Good Body, pero también a través de la gestualidad y el uso del espacio por parte de la(s) performer(s) dentro de lo que Boal llamaría el "espacio estético". En Fur is Back, por ejemplo, Ensler escenifica el derecho a la ira femenina presentando a una mujer que se ha convertido en una presencia incómoda en las fiestas de la "gente guapa" de su ciudad: mientras otras muestran su fascinación porque las pieles vuelven a estar de moda (de ahí el título), el personaje de Ensler está "fucking angry" por la situación de sus iguales en lugares como Afganistán bajo el yugo talibán, África en general con el cuchillo como espada de Damocles sobre sus clítoris, Congo en particular con las guerrillas violando por doquier o Pakistán con el ácido amenazando sus rostros. En la puesta en escena su cuerpo muestra la rabia ya no contenida, el grito contra la violencia machista convertido en gesto 'performativo', entendido este

\footnotetext{
${ }^{61}$ ENSLER 2001: 44.

${ }^{62}$ ENSLER 2001: 91.

${ }^{63}$ ENSLER 2001: XV.

Futhark 7 (2012)

Fernández, Más allá de los cuerpos dóciles, 131-161
}

ISSN 1886-9300 
término en el sentido que le da Fischer-Lichte ${ }^{64}$ : "I am on the floor", explica, "on the wall-to-wall plush carpet near the buffet table with its goat cheese quesadillas and grilled shrimp and chocolate martinis. I am on the floor screaming, stop it, stop it" ${ }^{65}$.

La toma de posesión de una voz personal y decidida por parte de las mujeres es también evidente en la última obra de Ensler publicada hasta la fecha, I Am an Emotional Creature, que ha pasado a formar parte de los eventos del Día-V en su iniciativa $V$ Girls y donde los sujetos activos son las niñas y mujeres jóvenes que habían ocupado sólo un lugar marginal en piezas anteriores, como hemos visto ya en algunos ejemplos. En esta pieza, reforzando las ideas sobre el potencial de rebeldía contra la docilidad de Foucault y Boal, se hace un llamamiento explícito a las adolescentes para que cambien el verbo "complacer (a los demás)" por conceptos como "atreverse" o "comprometerse" y para que tomen conciencia de los abusos ejercidos (por otros/as o por sí mismas a partir de mecanismos de asimilación de la violencia simbólica) sobre sus cuerpos. Como afirma Carol Gilligan en su introducción al libro, Ensler "begins with an introduction that is at once a cri de coeur and a call to action. [...] The monologues in this book are scripts for girls' resistance" ${ }^{66}$. Para promover esa resistencia activa, una vez más Ensler combina la visibilización que lleva a la denuncia con ejemplos de subversión concretos. Así, se muestran situaciones como la anorexia, los embarazos prematuros, la mutilación genital, las adicciones, la cirugía estética aplicada a menores o las agresiones en parejas adolescentes. Se incluyen también como temas de los

64 En su volumen ya citado de 2008, Erika Fischer-Lichte construye su conceptualización de performance a partir de las teorías de John L. Austin y Judith Butler. En el marco de este trabajo podemos decir que se trata de eventos teatralizados donde -como en los actos de habla 'performativos' de Austin- hay un proceso auto-referencial y constitutivo, porque el propio evento hace realidad la circunstancia social a la que se refiere (en este caso, el rechazo femenino a la violencia, que existe dentro y fuera del escenario). Además, añadiendo la perspectiva feminista en la línea de Butler, son puestas en escena en las que la identidad de género se va creando a medida que se actúa (en el ejemplo de Fur Is Back y otros textos de Ensler, la identidad de una mujer que, como todas las del mundo, carga por su condición con la amenaza de agresión sexista, y así lo manifiesta, entre otras formas, a través de su cuerpo).

${ }^{65}$ ENSLER 2007: 178.

66 Gilligan, Carol, "Foreword", en ENSLER, Eve, I Am an Emotional Creature. The Secret Life of Girls around the World, Nueva York: Villard, 2010, págs. xiii y xv.

Futhark 7 (2012) Fernández, Más allá de los cuerpos dóciles, 131-161

ISSN 1886-9300 
monólogos la trata de niñas, el abuso laboral o el reclutamiento forzoso, todo ello desde una perspectiva global que se apuntaba en The Vagina Monologues y The Good Body y que aquí toma una forma mucho más concreta. En esta pieza hablan muchachas estadounidenses de los barrios residenciales y del centro de la ciudad ("Let Me In", "Bad Boys"), niñas egipcias, francesas e iraníes ("Don't", "Sophie et Apolline", "The Joke about my Nose") o jóvenes prostituidas o vendidas en Bulgaria, Congo y Kenia ("I Have 35 Minutes Before He Comes Looking for Me", "A Teenage Girl's Guide to Surviving Sex Slavery", "Five Cows and a Calf"), explotadas en China ("Free Barbie") y atrapadas en el fuego cruzado entre Israel y Palestina ("The Wall" y "Sky Sky Sky").

Con su amplio ángulo de observación de la realidad de estas jóvenes desde una óptica feminista, el collage de testimonios que constituye I Am an Emotional Creature proporciona ejemplos específicos de resistencia de aquéllas que han dejado de ser víctimas de la aprendida docilidad 'foucaultiana', convirtiéndose en lo que Ensler llama refusers ("las que se niegan") ${ }^{67}$. Se compilan por ejemplo detalles que las entrevistadas aprecian de su físico (las curvas, la sonrisa, los hoyuelos en las mejillas...) y se permite a los personajes a quienes interpreta la performer reivindicar el placer, el control sobre su propio cuerpo y la capacidad de decidir: "my short skirt and everything under it", acaba uno de los monólogos, "is mine, mine, mine" ${ }^{68}$. Como ya hiciera en la conclusión de The Good Body, la autora insiste aquí en la sororidad y la potencial fuerza colectiva de las chicas, utilizando el plural "nosotras" para fomentar la identificación con los casos de rebelión que incluye en el texto:

From the Lebanese mountains

to the Kenyan village of El Doret

we are practising self-defense.

Versed in karate, tai chi, judo, and kung fu

we are no longer surrendering to our fate.

Now we are the ones who walk our girlfriends home

from school.

And we don't do it with macho. We do it with cool.

\footnotetext{
${ }^{67}$ ENSLER 2010: 107.

${ }^{68}$ ENSLER 2010: 125.

Futhark 7 (2012)

Fernández, Más allá de los cuerpos dóciles, 131-161
}

ISSN $1886-9300$ 
Our mothers are the Pink Sari Gang

fighting off the drunken men

with rose-pointed fingers and sticks $[\ldots]$

The Peshmerga women

in the Kurdish mountains with barrettes in their hair [...]

We are the Nigerian women

babies strapped to our backs

occupying the oil terminals of Chevron.

We are the women of Kerala

who refused to let Coca-cola

privatize our water.

We are Cindy Sheenan showing up in Crawford without a plan. $[\ldots]$

We are the girls who stopped cutting ourselves to release the

pain

and we are the girls who refused to have our clitoris cut and give up our pleasure. [...]

We are the women riding the high seas to offer needy women abortions on ships.

We are women documenting the atrocities

in stadiums with video cameras underneath our burqas.

We are seventeen and living for a year on a tree

and lying down to protect wild oaks.

We are out at sea interrupting whale murders.

We are freegans, vegans, trannies,

but mainly we are refusers.

We don't accept your world

your rules your wars.

We don't accept your cruelty and unkindness.

We don't believe some need to suffer for others to survive

or that there isn't enough to go around

or that corporations are the only and best economic arrangement.

And we don't hate boys, okay?

that's another bullshit story. $[\ldots]^{69}$

${ }^{69}$ ENSLER 2010: 107, 109 y 110.

Futhark 7 (2012) Fernández, Más allá de los cuerpos dóciles, 131-161

ISSN 1886-9300 


\section{Conclusiones}

Termino con esta cita empoderante el repaso a los puntos de contacto entre la praxis de Eve Ensler a través de sus obras dramáticas y de la iniciativa Día-V y el marco del Teatro de los Oprimidos de Augusto Boal, ambos construidos alrededor de las ideas de acción y de cambio. Como afirma Ann E. Armstrong y como espero haber demostrado con los ejemplos de los textos seleccionados, "[i]mplicit within TO [Theatre of the Oppressed] and feminism is the understanding that opression can be transformed and that victimhood is not inevitable" ${ }^{\prime 70}$. El teatro de Ensler está claramente dentro de ese proyecto feminista que pretende desterrar la victimización y fomentar la transformación social, política y cultural. Según describe su página web, el Día-V se apoya en tres ideas fundamentales:

1) Que el arte tiene el poder de llegar a la gente y de transformarla e inspirarla para actuar.

2) Que las mujeres empoderadas son líderes imparables y el cambio social y cultural se expande a través de su experiencia.

3) Que no se puede acabar con la violencia contra las mujeres sin tener en cuenta la intersección entre pobreza, racismo, medio ambiente y guerra ${ }^{71}$.

Para desarrollar esta filosofía, muy cercana a la de Augusto Boal y los laboratorios del Teatro de los Oprimidos que hay ya en muchos países, Ensler construye textos y puestas en escena que, en la línea del pensamiento 'foucaultiano' tardío sobre la posibilidad de rebelión contra el poder establecido, recogen experiencias de mujeres y chicas muy diferentes que se han visto afectadas por políticas culturales y prácticas sociales discriminatorias ejercidas sobre sus cuerpos. A partir de testimonios de agresión, abuso y violencia se denuncia el machismo imperante en la mayor parte de las sociedades del mundo, y se procura luego proporcionar ejemplos de resistencia que inspiren a las participantes en el hecho teatral para su propia vida. La idea, como en los ejercicios dramatúrgicos de Boal, es pasar de lo individual a lo colectivo a través de un

\footnotetext{
${ }_{71}^{70}$ ARMSTRONG 2006: 179.

${ }^{71}<$ www.vday.org/our-work>. Fecha de acceso: 21 de abril de 2011.

Futhark 7 (2012) Fernández, Más allá de los cuerpos dóciles, 131-161
}

ISSN 1886-9300 
proceso de identificación y toma de conciencia que Ensler cree que se da en muy pocos foros de interacción, siendo uno de ellos el que el brasileño llamaría el "espacio estético":

Theater insists that we inhabit the present tense -not the virtual tense or the politically correct tense. Theater demands that we truly be where we are. By being there together, we are able to confront the seemingly impossible, we are able to feel that which we fear might destroy us -and we are educated and transformed by that act.

Theater is sacred because it allows us, it encourages us, as a community of strangers, to go someplace together and face the issues and realities we simply cannot face alone. Alone, we are powerless, translating our suffering and struggle into our own private narcissistic injuries. When we become a group, these issues become social or political concerns, responsibilities, a reason for being there together ${ }^{72}$.

Diluyendo las barreras tradicionales entre el teatro y la realidad, entre lo visto/oído y lo hecho/practicado, tanto Ensler como Boal fomentan la iniciativa y el activismo en el público, que puede pasar -en distintos niveles y formas- de espectador a "espect-actor". Se convierte así idealmente a la "audiencia" en "agencia" contra la desigualdad en general y, en el caso del Día-V, específicamente contra una de sus manifestaciones más agudas: la violencia de género, una amenaza global para las mujeres. Porque como la propia Ensler afirma, "if you're willing to live with it, go through it, it doesn't own you. It's when you fear and resist and keep a reality away that it begins to own you"73. Se trata, pues, de enfrentar la situación para desterrar el miedo y la docilidad aprendidos individualmente, y de poner en común las injusticias para hacerlas visibles, denunciarlas, luchar contra ellas $y$, quién sabe, quizá terminar por convertirlas únicamente en algo que un día vimos sobre el escenario.

\section{REFERENCIAS BIBLIOGRÁFICAS}

\footnotetext{
${ }^{72}$ ENSLER, Eve, Insecure at Last. A Political Memoir, Nueva York, Villard, 2008 (2006), pág. 75.

${ }^{3}$ GREENE 2001: 161.

Futhark 7 (2012)

Fernández, Más allá de los cuerpos dóciles, 131-161
}

ISSN $1886-9300$ 
ARMSTRONG, Ann Elizabeth, "Negotiating Feminist Identities and Theatre of the Oppressed", en COHEN-CruZ, Jan; SchuTZMAn, Mady (eds.), A Boal Companion. Dialogues on Theatre and Cultural Politics, Nueva York y Londres, Routledge, 2006, págs. 173-184.

BeAusoleIL, Natalie, "Speaking the Unspeakable: An Interview with the Producers of The Vagina Monologues in St. John's, Newfoundland", en Atlantis, 27.2, 2003, <http://forms.msvu.ca/atlantis/vol/272all/272beausoleil.pdf>. Fecha de acceso: 14 de marzo de 2011.

BeLL, Susan E.; ReVerby, Susan M., "Vaginal Politics: Tensions and Possibilities in The Vagina Monologues", en Women's Studies International Forum, 28, 2005, págs. 430-444.

BOAL, Augusto, Theatre of the Oppressed, Nueva York, Theatre Communications Group, 1985 (1974).

---, Legislative Theatre. Using Performance to Make Politics, Londres y Nueva York, Routledge, 2005 (1998).

---, The Rainbow of Desire. The Boal Method of Theatre and Therapy, Londres y Nueva York, Routledge, 2006a (1995).

---, The Aesthetics of the Oppressed, Londres y Nueva York, Routledge, 2006b.

Bourdieu, Pierre; Wacquant, Loïe, Réponses. Pour une anthropologie réflexive, París, Seuil, 1992.

DanAHER, Geoff, Tony Schirato; WeBB, Jen, Understanding Foucault, Londres, Sage, 2000.

DIAMOND, Irene; QUINBY, Lee, "Introduction", en DIAMOND, Irene; QUINBY, Lee (eds.), Feminism \& Foucault. Reflections on Resistance, Boston, Northeastern University Press, 1988, págs. ix-xx.

EnSLeR, Eve, The Vagina Monologues, Nueva York, Villard, 1998.

---, The Good Body, Londres, William Heinemann, 2001.

Futhark 7 (2012) Fernández, Más allá de los cuerpos dóciles, 131-161

ISSN 1886-9300 
---, Fur Is Back, en EnSLER, Eve (ed.), A Memory, A Monologue, A Rant, and a Prayer. Writings to Stop Violence against Women and Girls, Nueva York, Villard, 2007, págs. 176-179.

---, Insecure at Last. A Political Memoir, Nueva York, Villard, 2008 (2006).

---, I Am an Emotional Creature. The Secret Life of Girls around the World, Nueva York, Villard, 2010.

ENSLER, Eve; Tenneson, Joyce, Vagina Warriors, Nueva York y Boston, Bulfinch Press, 2005.

FERNANDEZ, J. Manuel, "La noción de violencia simbólica en la obra de Pierre Bourdieu: una aproximación crítica", en Cuadernos de Trabajo Social, 18, 2005, págs. 7-31.

Fernández Morales, Marta, "When Theater Becomes a Crusade against Violence", en BAHUN-RADUNOVIĆ, Sanja; RAJAN, V.G. Julie (eds.), Violence and Gender in the Globalized World. The Intimate and the Extimate, Aldershot y Burlington, Ashgate, 2008, págs. 205-221.

FISCHER-LICHTE, Erika, The Transformative Power of Performance. A New Aesthetics, Londres y Nueva York, Routledge, 2008.

Gilligan, Carol, "Foreword", en ENSLER, Eve, I Am an Emotional Creature. The Secret Life of Girls around the World, Nueva York, Villard, 2010, págs. xiii-xvii.

Greene, Alexis, Women Who Write Plays. Interviews with American Dramatists, Hanover, Smith \& Kraus, 2001.

KATTWINKEL, Susan. "Spreading American Feminism. The Vagina Monologues and Cultural Identity", en MAUFORT, Marc y DE WAGTER, Caroline (eds.), Signatures of the Past. Cultural Memory in Contemporary Anglophone North American Drama, Bruselas, Peter Lang, 2008, págs. 249-257.

LACY, Suzanne, "Activism in Feminist Performance Art", En COHENCruz, Jan; SchutzMAN, Mady (eds.), A Boal Companion. Dialogues on Theatre and Cultural Politics, Nueva York y Londres, Routledge, 2006, págs. 91-102.

Futhark 7 (2012) Fernández, Más allá de los cuerpos dóciles, 131-161

ISSN $1886-9300$ 
MCNAY, Lois, Foucault \& Feminism: Power, Gender and the Self, Boston, Northeastern University Press, 1992.

RenshaW, Sal. "Divine Gifts and Embodied Subjectivities in The Vagina Monologues", en International Feminist Journal of Politics, 6.2, junio 2004, págs. 318-325.

Steinem, Gloria, "Foreword", en ENSLER, Eve, The Vagina Monologues, Nueva York, Villard, 1998, págs. vii-xvii.

Futhark 7 (2012) Fernández, Más allá de los cuerpos dóciles, 131-161

ISSN $1886-9300$ 